Editorial

\title{
International Journal of Structural Glass and Advanced Materials Research: A New Open Platform for Materials Science
}

\author{
${ }^{1}$ Chiara Bedon, ${ }^{2}$ Mario D'Aniello, ${ }^{3}$ Andreas Luible and ${ }^{4}$ Flavio Stochino \\ ${ }^{1}$ Department of Engineering and Architecture, University of Trieste, Trieste, Italy \\ ${ }^{2}$ Department of Structures for Engineering and Architecture, University of Naples Federico II, Naples, Italy \\ ${ }^{3}$ Competence Center Façade and Metal Engineering, Lucerne University of Applied Sciences and Arts, \\ Lucerne, Switzerland \\ ${ }^{4}$ Department of Civil Environmental Engineering and Architecture, University of Cagliari, Cagliari, Italy
}

\section{Introduction}

\section{Editorial}

The International Journal of Structural Glass and Advanced Materials Research (IJSGAMR) is a new peer-reviewed, open access journal, which covers all aspects of theoretical and practical research of materials science. The journal aims to promote international exchange of knowledge and broad discussion on advancements, outcomes and recent developments in materials research for engineering applications.

In the last years, the development of new materials and relevant technologies (i.e., industrial processes as well as constructional requirements) has been an ongoing challenge for researchers and engineers to answer to current societal demands: Energy savings, urban resilience and smart cities. In particular, buildings and civil infrastructures are very complex systems, in which single components are strongly linked to each other and their own actual performance/value (including structural, architectural, thermal aspects, etc) is actually affected by reciprocal interactions of single parts belonging to a complex assembly. In this view, buildings can represent both energy consumers and sources (thanks to solar cells or other renewable energies), while the infrastructures provide connections and information to this system. Nevertheless, given that both economical and environmental resources are limited and should be preserved, sustainable and resilient design solutions are unavoidable as well as necessary.

It is clear that the knowledge of the mechanical and durability features of constructional materials, as well as the related technologies, should be enhanced in order to allow the designers to control and effectively conceive the structural behavior, but even the infrastructure owners to plan the maintenance scenarios throughout the entire life cycle considering the potential impacts on the environment. Novel studies on recycled concrete have shown, for example, that the latter can be designed and produced reducing the environment pollution, without affecting its usual structural performances. On the other hand, nowadays metallurgical technology is developing even more challenging metal alloys reducing the emission of equivalent $\mathrm{CO}_{2}$ and minimizing the energy consumptions.

Nowadays, at the same time, the issue of structural safety under natural (e.g., seismic events, floods, landslides, etc.) and anthropic (e.g., blast, fire, impacts, etc.) exceptional loading scenarios is an urgent criticism still far from being solved. Indeed, examples of extreme loading scenarios are even more frequent. The tragic news of the terrorist attacks of recent years raise important questions regarding the real safety and reliability of existing and new buildings. In addition, often an explosion is followed or anticipated by fire. In these conditions, where materials and structures are pushed to their limits, the high performance features of new materials can represent a smart contribution to the design of robust buildings or to retrofit existing structural systems.

In this framework, the use of structural glass represents a key example. Originally considered for nonstructural and secondary components in buildings only, glass has been strongly used in the last decades to ensure transparency, for windows and cladding walls. Despite glass is a well-known brittle material with limited tensile resistance, several studies and design applications proved that - especially once combined with other materials (e.g., steel, Fiber-Reinforced-Polymers, timber, etc.) - glass can offer very interesting structural performances and act as a novel constructional material. Hybrid glass systems have been successfully used as load-carrying components, in the form of beam, fins and columns, as well as in even geometrically complex 
façades, cladding walls, roofs, stairs. Interesting and promising safe design performances can be obtained also for blast-resistant glazing curtain walls, where most of the incoming energy can be dissipated by special connectors. Laminates and meta-materials are other examples of engineering answer to rising structural demands. The global structural behavior of laminates depends on the lamination stacking sequence, while the microstructure or lattice of meta-materials can provide a tuned property on the macro-scale. For example, it is possible to design materials with negative Poisson's ratio or negative density. In addition, very promising results have been obtained for the so-called invisibility cloak that can shelter structures from earthquake or provide isolation from wave in a specific frequency range.

Metal alloys are other effective examples of challenging materials that have great impact on the market of constructions in terms of mechanical properties, recyclability and sustainability, optimization of constructional processes and limitation of related costs. For example, metal foams (i.e., cellular materials consisting of a solid metal with gas-filled pores comprising a large portion of the volume) are becoming very popular to anti-impact and/or anti-blast retaining systems, but some applications for composite deck with steel girders are also proposed with very promising spinoff. The metallurgic industry is also promoting and developing high performance steels, which guarantee very effective mechanical features (e.g., high yield stress, high toughness at low temperature, high ductility, low residual stress, etc.). The potential use of this class of steels ranges from high-rise tower, mega-structures, long span bridges to innovative fasteners. However, applications in seismic areas are very promising, because the use of high strength materials for non-dissipative members is effective to guarantee the fulfillment of the hierarchy of resistance. On the other hand, some interesting novel alloys (e.g., either steel or aluminum based) characterized by very low yield stress but high ductility are becoming very popular to design dissipative components in structural systems as well as anti-seismic devices based on the hysteretic dissipation. It is also worth noting that novel metal alloys are recently adopted for civil constructions. Indeed, brass has been recently used for dissipative devices due to its high friction coefficient. Titanium is widely used for aerospace structures, but recently some interesting applications in civil engineering are under development due to its resistance against corrosion and its stability at high temperature. Other examples of metal alloys with a wide range of application are the Shape-Memory Alloys (SMA). SMA is an alloy that recover its original shape after applied loads are removed. These lightweight materials are adopted in robotics, automotive, aerospace, biomedical industries and recently also in civil engineering for anti-seismic devices.

All themes related to the above mentioned and others innovative materials will be covered by the International Journal of Structural Glass and Advanced Materials Research. In this regard, careful consideration is paid especially for theoretical, experimental and numerical investigation on novel solutions, including structural glass as well as hybrid systems, fiber-reinforced pultruded composites, laminates, polymers, metal alloys, high performance (both high and low strength) steels and shape memory alloys. Coverage also includes (but is not limited to): Design philosophy and safety concepts for extreme loading conditions, strength and stability, structural innovation, sustainability and architectural aspects, thermal performance of civil engineering materials, fabrication techniques relevant to the application of advanced materials as load-bearing components or assemblies in buildings.

The International Journal of Structural Glass and Advanced Materials Research aims to serve as an effective platform and key resource for the promotion of scientific and technical exchange between academic scientists, professional engineers, designers. In this perspective, International Journal of Structural Glass and Advanced Materials Research publishes high-quality research, including full-length original papers, as well as review articles, case studies, technical notes and short research letters. To this aim, all papers are subjected to blind review process by a distinguished team of international experts.

On behalf of the Editorial Office and Editorial Board, we invite all Authors and Reviewers and thank them for the valuable contributions that they are going to give to the Journal of Structural Glass and Advanced Materials Research.

We believe that we can develop the International Journal of Structural Glass and Advanced Materials Research as a premier interdisciplinary open platform for materials science.

The Editor-In-Chief and the Associate Editors. 